\title{
Tratamento ortodôntico-cirúrgico de má oclusão classe III: relato de caso clínico
}

\section{Class III maloclusion orthodontic-surgical treatment: clinical case report}

\section{Tratamiento ortodóntico-quirúrgico de maloclusión clase III: relato de caso clínico}

\author{
Wilder Javier Baldiviezo Cáceres ${ }^{1}$ \\ Angie Patricia Castro Merán \\ Daniel Salvatore de Freitas ${ }^{2}$ \\ Karina Maria Salvatore Freitas iD \\ 1 wilder5_4@hotmail.com \\ 2 institutofreitas@gmail.com \\ Endereço para correspondência: \\ Karina Maria Salvatore Freitas \\ Instituto Freitas de Odontologia \\ Rua Gérson França, 9-02 \\ Vila Mesquita \\ 17015-200 - Bauru - São Paulo - Brasil \\ E-mail:kmsf@uol.com.br
}

Recebido: 03.08.2020

Modificado: 24.08.2020

Aceito: 01.09.2020

\section{RESUMO}

Relatar caso clínico de um paciente com maloclusão Classe III que tinha sido compensado ortodonticamente e que depois optou por realizar tratamento cirúrgico. Foi realizado o tratamento ortodôntico de descompensação para melhorar as inclinações dos incisivos superiores e inferiores. O caso foi planejado virtualmente por meio de software para simular tridimensionalmente o avanço maxilar e retração mandibular. Após a geração e impressão do guia cirúrgico, foi realizado o tratamento cirúrgico sob anestesia geral. Após o procedimento cirúrgico o paciente apresentou recuperação satisfatória, fazendo uso de aparelho para finalização ortodôntica. Após 5 meses de pós-operatório o aparelho ortodôntico foi removido para entrar na fase de contenção. No controle de 1 ano e 3 meses observou-se uma manutenção da estabilidade oclusal sem presença de recidiva. Ao controle radiográfico observou-se uma consolidação e remodelação óssea completa. O paciente apresentou apresentando um perfil característico de paciente Classe I. A combinação dos tratamentos ortodôntico e cirúrgico foram efetivos para correção da discrepância esquelética maxilomandibular do paciente que apresentava má oclusão de Classe III.

PALAVRAS-CHAVE: Ortodontia. Cirurgia ortognática. Má-oclusão. 
opted for surgical treatment. Orthodontic decompensation treatment was performed to improve the inclinations of the upper and lower incisors. The case was planned virtually through software to simulate three-dimensional maxillary advancement and mandibular retraction. After generating and printing the surgical guide, surgical treatment was performed under general anesthesia. After the surgical procedure, the patient presented a satisfactory recovery, using an appliance for orthodontic completion. After 5 months postoperatively, the orthodontic appliance was removed to enter the containment phase. In the control of 1 year and 3 months, a maintenance of occlusal stability was observed without the presence of recurrence. Radiographic control showed complete bone consolidation and remodeling. The patient presented with a characteristic Class I patient profile. The combination of orthodontic and surgical treatments was effective in correcting the maxillomandibular skeletal discrepancy of the patient who had Class III malocclusion.

KEYWORDS: Orthodontics. Orthognathic surgery. Malocclusion.

\section{RESUMEN}

Reportar un caso clínico de un paciente con maloclusión Clase III que había sido compensado ortodónticamente y que posteriormente optó por tratamiento quirúrgico. Se realizó un tratamiento de descompensación ortodóncica para mejorar las inclinaciones de los incisivos superiores e inferiores. El caso se planificó virtualmente mediante software para simular el avance del maxilar y la retracción mandibular en tres dimensiones. Luego de generar e imprimir el guía quirúrgico, se realizó el tratamiento quirúrgico bajo anestesia general. Tras el procedimiento quirúrgico, el paciente presentó una recuperación satisfactoria, utilizando un aparato de ortodoncia. Después de 5 meses de posoperatorio, se retiró el aparato de ortodoncia para entrar en la fase de contención. En el control de 1 año y 3 meses se observó un mantenimiento de la estabilidad oclusal sin presencia de recidiva. El control radiográfico mostró consolidación y remodelación ósea completa. El paciente presentaba un perfil de paciente característico de Clase I. La combinación de tratamientos ortodóntico-quirúrgico fue eficaz para corregir la discrepancia esquelética maxilomandibular del paciente que tenía maloclusión de Clase III.

PALABRAS CLAVE: Ortodoncia. Cirugía ortognática. Maloclusión. 


\section{INTRODUÇÃO}

As más oclusões de Classe III esqueléticas podem apresentar como características estruturais a retrusão maxilar, a protrusão mandibular ou uma associação de ambas. Entre seus principais fatores etiológicos, destacam-se o padrão genético (predominante) e o crescimento ósseo aposicional ${ }^{1}$.

Há, também, uma correlação entre a respiração bucal e a atrésia maxilar durante a fase de crescimento, pela posição mais inferior da língua, que deixa de exercer a pressão necessária ao desenvolvimento maxilar normal, o que pode estar presente em casos de Classe III. Entre as características faciais comuns de pacientes Classe III de Angle, podem-se destacar: o perfil facial côncavo, a proeminência do terço inferior da face e a projeção do lábio inferior em relação ao lábio superior ${ }^{2}$.

$\mathrm{O}$ tratamento das deformidades dentofaciais é baseado em cinco princípios básicos: harmonia facial, harmonia dentária, oclusão funcional, saúde das estruturas orofaciais e estabilidade do procedimento. Alguns casos podem ser compensados ortodónticamente, no sentido de camuflar a má oclusão ${ }^{3}$.

As deformidades dentofacias podem ser tratadas por meio de procedimentos cirúrgicos isolados na mandíbula ou na maxila, ou de forma combinada. Cada tipo de deformidade esquelética requer um planejamento cirúrgico específico, que envolve o reposicionamento de segmentos do esqueleto facial por meio de osteotomias e da aplicação de fixação interna estável. Dessa forma, com base em fundamentos clínicos, biológicos, estéticos e biomecânicos, se justifica a correta indicação da cirurgia ortognática proporciona a função adequada do sistema musculoesquelético, harmonia facial e estabilidade em longo prazo ${ }^{4}$.

O objetivo deste trabalho é relatar um caso clínico de um paciente que apresentava uma má oclusão de Classe III e em quem foi realizado tratamento ortodôntico-cirúrgico. A hipótese deste trabalho foi que a combinação de ortodontia e cirurgia ortognática é efetiva para correção da discrepância esquelética maxilomandibular, que era a queixa principal do paciente.

\section{RELATO DE CASO}

Paciente masculino, de 23 anos e 6 meses, leucoderma, compareceu à consulta relatando queixa estética de assimetria facial, principalmente de perfil.

$\mathrm{O}$ paciente tinha iniciado o tratamento ortodôntico durante um ano com aparelho móvel aos 13 anos de idade. Passou a utilizar aparelho fixo durante 4 a 5 anos, para compensação e contenção durante mais um ano. O paciente relatou recidiva da má oclusão, razão pela qual voltou a utilizar aparelho fixo durante mais 3 anos e mais um ano com contenção móvel (6 meses contínuo e 6 meses só durante a noite). Após algum tempo do tratamento, relatou dor na ATM, dor de ouvido e retração gengival. Depois de procurar informações sobre cirurgia ortognática, chegou à consulta no Instituto Freitas de Odontologia.

Ao exame clínico intraoral, o paciente foi diagnosticado com uma relação molar de Classe III bilateral, sendo completa do lado direito e $1 / 2$ do lado esquerdo. Relação de caninos de $1 / 2$ Classe III do lado direito e de Classe I do lado esquerdo.

Ao exame clínico extraoral apresentava um perfil côncavo, característico de um padrão facial Classe III. Sem desvios de linha média facial nem assimetrias dignas de nota (Figura 1). Foi realizado exame radiológico (Figura 2).
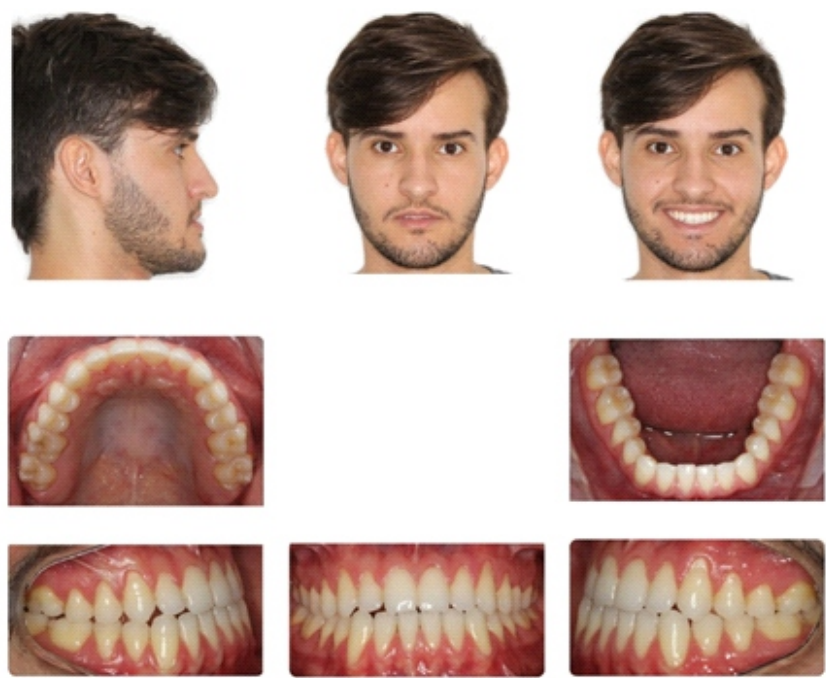

Figura 1 - Fotografias iniciais.
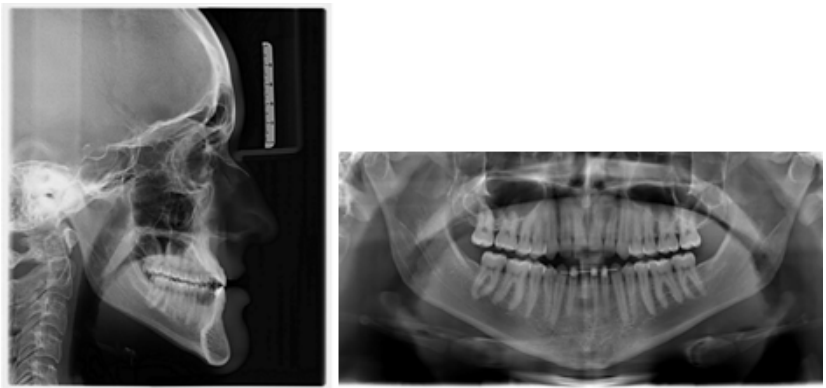

Figura 2 - Radiografias iniciais.

O traçado cefalométrico foi realizado utilizando o Software Dolphin Imaging seguindo a Análise de Arnett e Mclaughlin e sobrepondo a fotografia lateral do paciente na posição natural da cabeça. Foi diagnosticada uma retrusão maxilar e uma protrusão mandibular com os incisivos superiores ligeiramente vestibularizados $\left(52^{\circ}\right)$ e incisivos inferiores acentuadamente lingualizados $\left(85^{\circ}\right)$, resultado da compensação ortodôntica previa (Figura 3). 


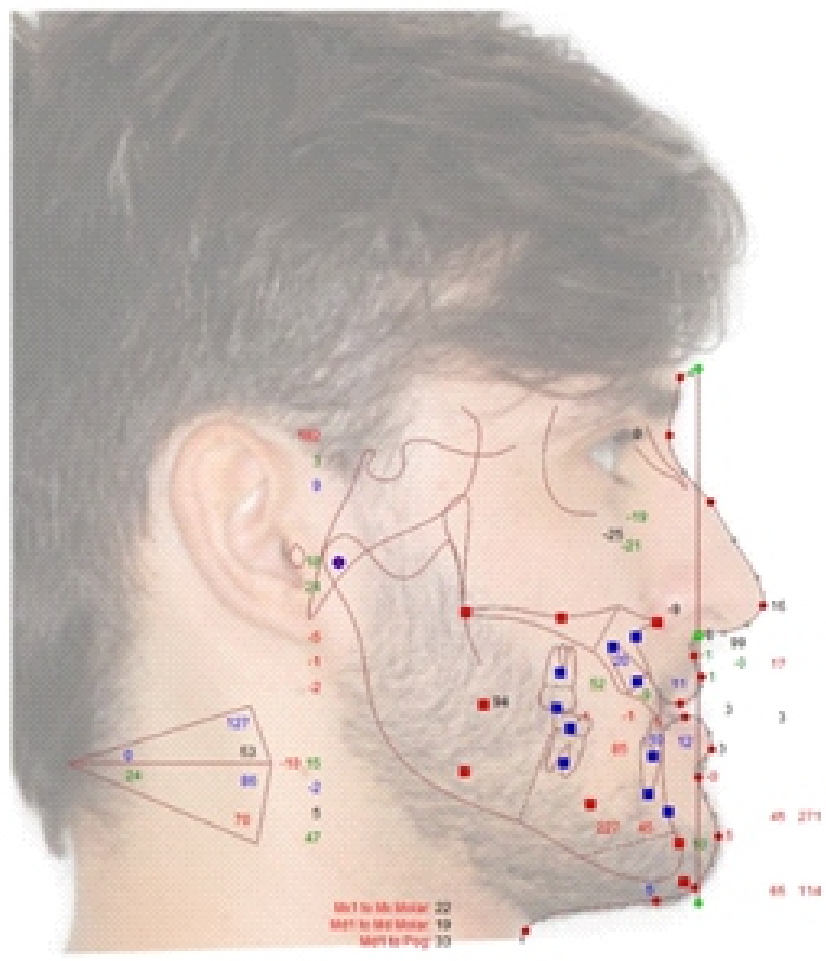

Figura 3 - Traçado cefalométrico com sobreposição da foto lateral, realizada no software Dolphin Imaging.

Foi planejado o ortodôntico, o qual incluiu uso de aparelho fixo, nivelamento e alinhamento, coordenação dos arcos dentários e uso de elásticos intermaxilares de Classe II para descompensação dentária, aumentando a severidade da Classe III para posterior realização da cirurgia ortognática bimaxilar. Após 6 meses e 20 dias de iniciada a descompensação com aparelho fixo, observou-se uma melhora nas inclinações dos incisivos com o uso do elástico de classe II e instalação de molas de secção aberta entre laterais e caninos superiores para correção de discrepância de Bolton. O caso foi avaliado novamente com 8 meses 10 dias. Notou-se inclinações dos incisivos em uma posição favorável, correção da discrepância de Bolton, alcançada mediante a abertura de espaço entre laterais e caninos (Figura 4). Neste ponto, o paciente encontrava-se pronto para a cirurgia.
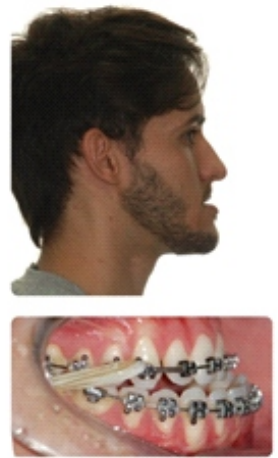
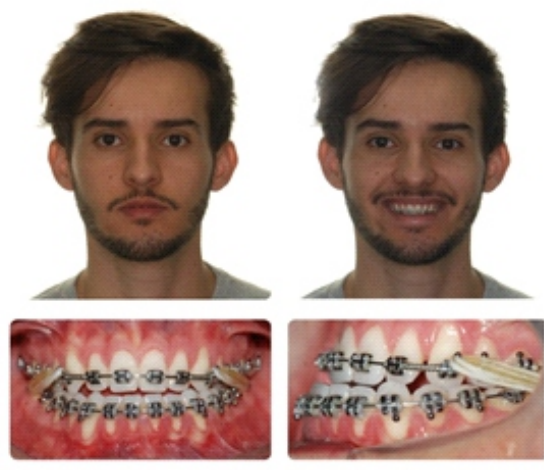

Figura 4 - Progresso 8 meses 10 dias (pronto para a cirurgia).
Foram realizadas também novas radiografias e fotografias, modelos de gesso com registro de cera na posição cêntrica para guiar o paciente durante a realização da tomografia computarizada e posteriormente escanear os modelos de gesso para montar o paciente virtual no software Dolphin Imaging e realizar o planejamento 2D e 3D do caso para confecção do guia cirúrgico. Foi feita a análise facial do paciente, preenchendo o formulário do exame facial desenvolvido por Arnett em 2003. Determinou-se que o paciente apresentava uma maxila retruída e uma mandíbula protruída em relação a LVV. Com exposição adequada dos incisivos e $1 \mathrm{~mm}$ de desvio da linha média à direita. Também realizado o traçado cefalométrico e sobreposição 2D no software Dolphin Imaging (Figura 5). Conseguiu-se pontuar os problemas a corrigir, obtendo também uma tabela com referência aos parâmetros alterados.
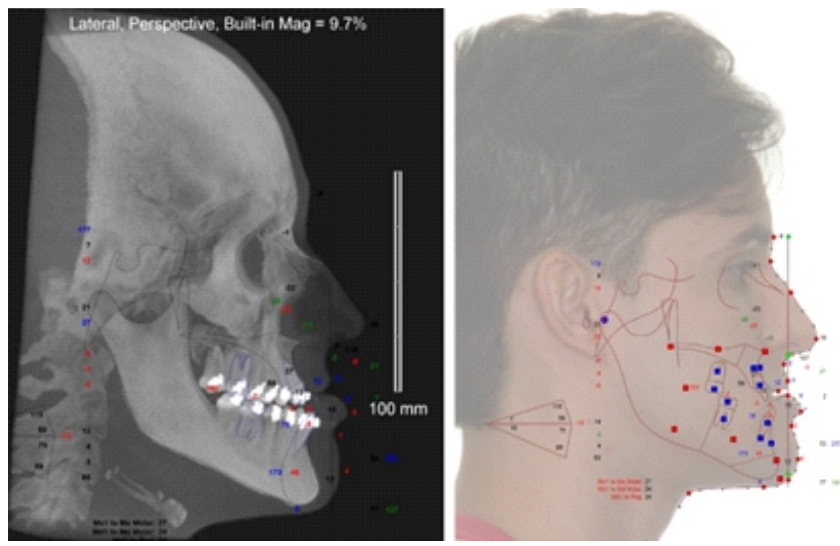

Figura 5 - Traçado cefalométrico e sobreposição.

Depois de montar o caso simulou-se as movimentações para obter um preditivo 2D do caso. Concluiu-se que seria necessário um avanço da maxila na região dos incisivos e recuo da mandíbula em todas as regiões, associado a uma rotação horária bimaxilar e sem mentoplastia.

Foi realizada a tomografia computadorizada sem fio ortodôntico para reduzir o artefato e utilizado um registro de cera na relação cêntrica. Também foram escaneados os modelos de gesso na oclusão inicial e final (Figura 6). Com essa documentação foi possível montar o crâneo composto do paciente para o planejamento 3D (Figura 7).

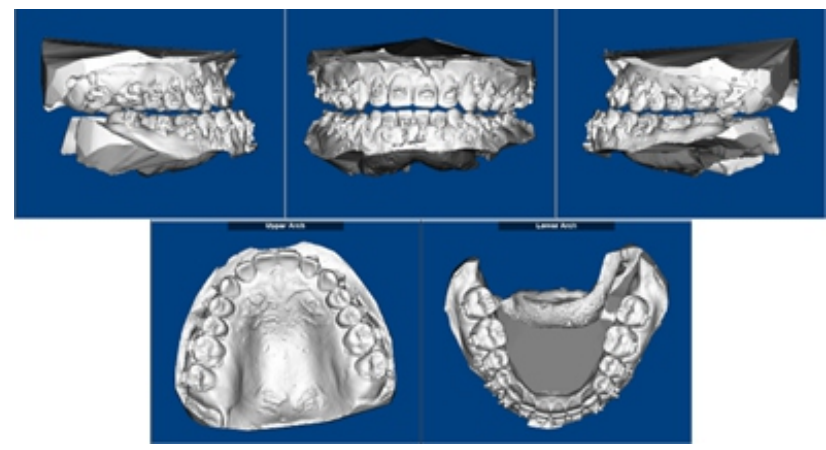

Figura 6 - Modelos de gesso escaneados. 


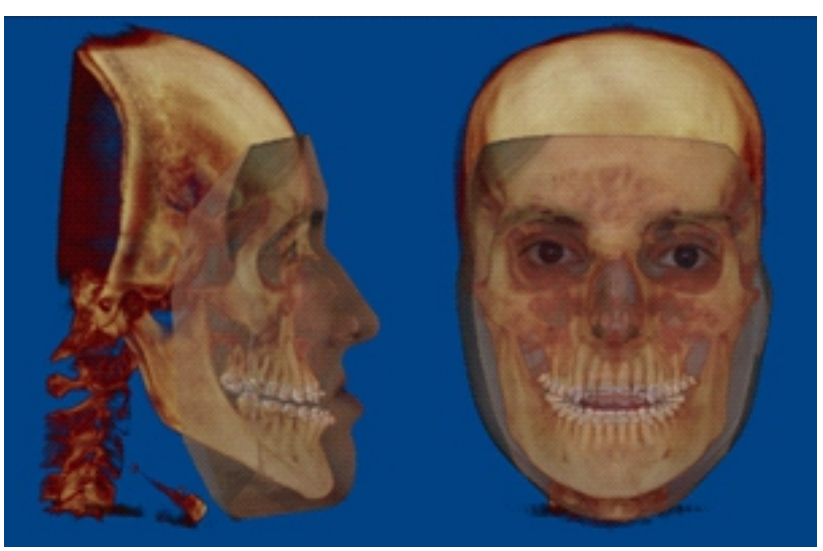

Figura 7 - Crânio composto em software Dolphin Imaging.

O planejamento cirúrgico final foi definido no software 3D baseado no planejamento $2 \mathrm{D}$ e na análise facial. No sentido anteroposterior: foi proposto um avanço maxilar na região dos incisivos $+1.40 \mathrm{~mm}, \mathrm{SNA}+4.97, \mathrm{SNP}+6.17$. $\mathrm{Na}$ mandíbula, uma retração de $-4.61 \mathrm{~mm}$ na região dos incisivos, - $4.99 \mathrm{~mm}$ na região do ponto $\mathrm{B}$ e $-5.38 \mathrm{~mm}$ na região do pogônio (Figura 8).

No sentido vertical: teve uma impactação pouco significativa dos incisivos superiores de $-0.23 \mathrm{~mm}$. Nos incisivos inferiores foi de $-4.8 \mathrm{~mm}$ por conta da correção do overbite negativo que ele apresentava (Figura 9). Foi gerado o protótipo do guia cirúrgico, com o objetivo de levar a mandíbula na posição final para depois colocar a maxila em oclusão (Figura 10).

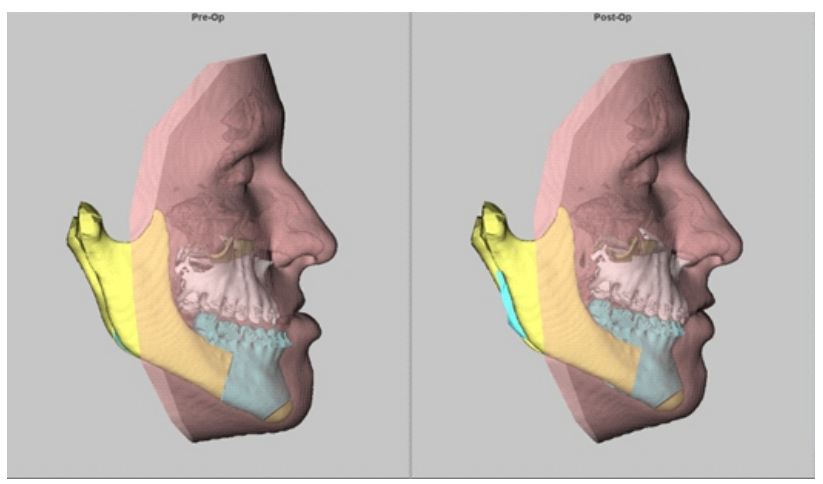

Figura 8 - Planejamento anteroposterior.

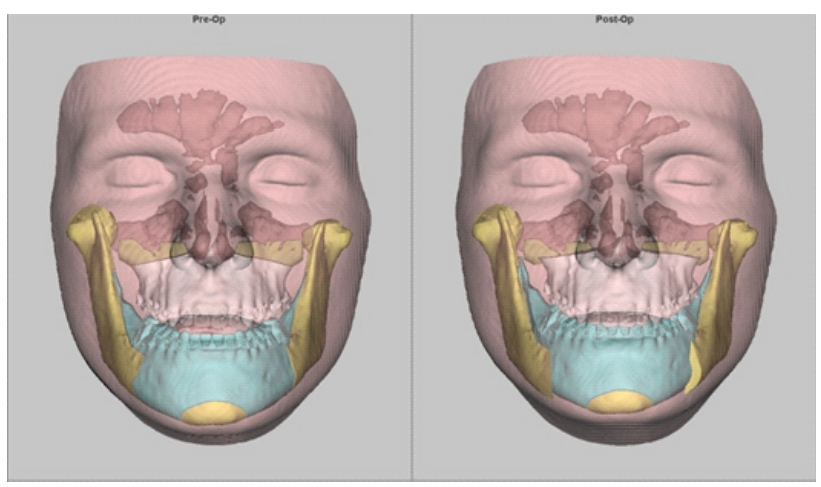

Figura 9 - Planejamento vertical e linha média.
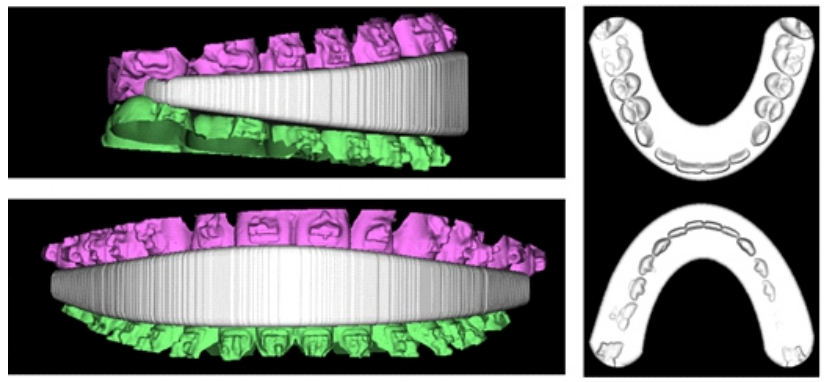

Figura 10 - Protótipo do guia cirúrgico.

O procedimento foi realizado sob anestesia geral com intubação nasotraqueal. Após a incisão e descolamentos foram expostos os ramos mandibulares bilaterais, as fraturas foram realizadas por meio de osteotomia sagital. Utilizou-se o guia cirúrgico para levar a mandíbula na posição planejada, estabilizada com bloqueio por elástico corrente, para realizar FIR dos ramos mandibulares. O avanço maxilar foi realizado com osteotomia tipo LeFort I, e FIR na máxima intercuspidação com a mandíbula. Para finalizar, foram realizadas suturas de base alar, maxila e mandíbula, remoção do TOF e bloqueio maxilomandibular com elásticos intermaxilares que serviram para ajudar na estabilização das osteotomias e também na melhora da intercuspidação dentária.

Aos 5 meses 5 dias de pós-operatório, o paciente estava sem presença de edema. Conseguiu-se o fechamento completo dos diastemas e uma correta intercuspidação com os caninos e molares em Classe I, permitindo a remoção do aparelho ortodôntico. Foi colocada também uma contenção $3 \times 3$ na arcada inferior, e uma placa removível (Hawley) para contenção superior. No controle de 1 ano 9 meses observou-se uma manutenção da estabilidade oclusal sem presença de recidiva (Figura 11). Ao controle radiográfico observou-se uma consolidação e remodelação óssea completa (Figura 12).
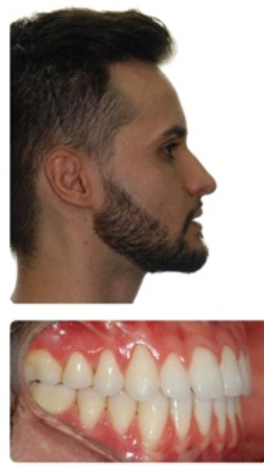
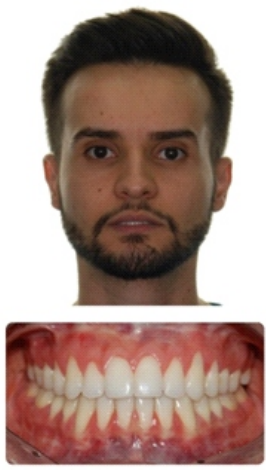
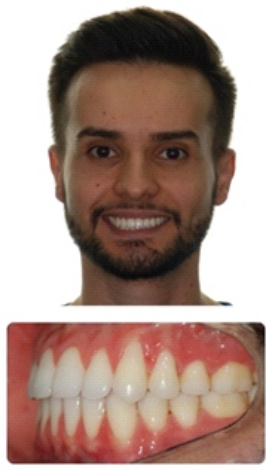

Figura 11 - Pós-operatório de 1 ano e 9 meses. 

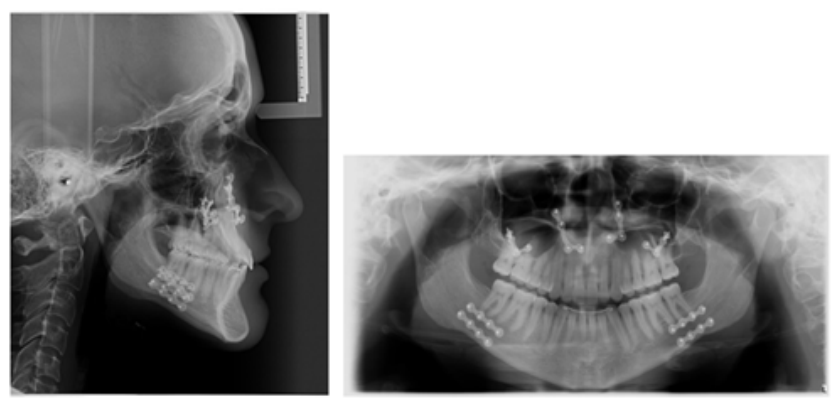

Figura 12 - Controle radiográfico após 1 ano e 9 meses.

\section{DISCUSSÃO}

A correção da má oclusão de Classe III esquelética é, geralmente, alcançada pela compensação dentária ou pelo tratamento ortodôntico-cirúrgico, sendo que a discrepância óssea influência na decisão pela cirurgia. Esta é necessária para que se atinjam resultados de alta qualidade, em termos de oclusão e estética ${ }^{5}$. Na má oclusão de Classe III, a compensação dentária geralmente evolui para a protrusão da dentição superior e inclinação lingual da dentição mandibular, que irá desenvolver menos aparência de Classe III para pacientes e maior tempo de tratamento (para descompensação dentária) com prognatismo esquelético classe III e mandibular. Provavelmente é uma desvantagem da abordagem convencional devido à menor satisfação do paciente ${ }^{6}$. Em este sem tido, um estudo brasileiro realizado sobre má oclusão de Classe III, a incidência de deformidades esqueléticas teve maior incidência em mulheres. Essa característica leva a problemas psicológicos, pois esses pacientes dão grande valor à sua aparência, e a melhora da estética é o grande motivador para a procura do tratamento ${ }^{7}$. Neste relato de caso, o paciente havia sido submetido a tratamento ortodôntico compensatório na adolescência e não se encontrava satisfeito com os resultados obtidos.

O plano de tratamento ideal deve ser formulado de forma a afetar os traços faciais na forma mais positiva, enquanto corrige a mordida ${ }^{8}$. O plano de tratamento deve ser ortodôntico ou cirúrgico ortodôntico, conforme determinado pelo exame facial. O movimento ortodôntico pode satisfazer a correção da mordida e a cirurgia pode ser do maxilar ou do maxilar e mandíbula. No caso de nosso paciente, foi realizada cirurgia bimaxilar, com um avanço do maxilar e recuo da mandíbula.

Os defeitos esqueléticos graves são tratados com protocolos cirúrgicos ortognáticos que incorporam tanto a ortodontia como a cirurgia. As intervenções ortodônticas pré e pós-operatórias e o planejamento cirúrgico baseados nas radiografias cefalométricas laterais pré-operatórias são fatores importantes para o sucesso desses tratamentos.
Alterações nas estruturas esqueléticas e dos tecidos moles após a cirurgia podem ser previstas usando traçados cefalométricos convencionais ou software de computador'. No caso apresentado, utilizamos o planejamento digital por meio do software Dolphin Imaging para a simulação das movimentações a serem realizadas.

O tratamento ortodôntico-cirúrgico da má oclusão de Classe III esquelética tem sido extensamente estudado. De forma geral, ele apresenta uma boa estabilidade em longo prazo e leva os pacientes a uma grande satisfação com a estética alcançada. As mudanças esqueléticas mais comumente relatadas na literatura após a correção ortodôntica-cirúrgica são o avanço maxilar e o retrocesso mandibular, enquanto as mudanças dentárias incluem a verticalização dos incisivos inferiores ${ }^{10}$. No caso apresentado após 1 ano do término do tratamento, observouse estabilidade das correções alcançadas e o paciente mostrou muita satisfação com os resultados estéticos.

A fase de preparo ortodôntico para a cirurgia é importante para o sucesso do caso, e o paciente encontra-se pronto para a cirurgia quando ambas as arcadas dentárias estão alinhadas e niveladas; nesse momento, os fios presentes devem ser retangulares 0.019 " x 0.025 " ou 0.021 " x $0.025 "$ ", para permitir a soldagem de ganchos que servirão de apoio para os elásticos intermaxilares ${ }^{11}$. No caso relatado neste estudo o preparo ortodôntico foi de vital importância para alinhar as arcadas antes do tratamento cirúrgico.

Convencionalmente, a oclusão dentária é determinada usando o modelo de cirurgia baseado no planejamento cefalométrico bidimensional para o movimento dos segmentos maxilar e mandibular. Posteriormente, placas cirúrgicas de acrílico são fabricadas no articulador e usadas em salas de cirurgia para reposicionar a maxila e a mandíbula $^{12}$. No caso do nosso paciente, os modelos de gesso foram escaneados para a realização do guia cirúrgico.

O planejamento da oclusão virtual para procedimentos cirúrgicos ortognáticos poderia reduzir os erros causados pela oclusão manual com modelos odontológicos, como transferência do arco facial, reposicionamento da mandíbula e movimento dos segmentos ósseos ${ }^{13}$.

A abordagem cirúrgica em primeiro lugar tornou-se um plano de tratamento popular para os pacientes em relação ao fator que pode diminuir a duração do tratamento pela redução da ortodontia pré-operatória. Essa abordagem pode levar a uma melhor cooperação e demonstra satisfação dos pacientes em relação à melhora imediata do perfil facial ou da constrição das vias aéreas superiores 14 . No caso apresentado, o paciente optou pela cirurgia ortognática mesmo depois de ter passado por um tratamento ortodôntico extenso, sendo sua queixa principal a estética facial. 


\section{CONCLUSÃO}

No caso clínico apresentado, a combinação dos tratamentos ortodôntico e cirúrgico foram efetivos para correção da discrepância esquelética maxilomandibular do paciente que apresentava má oclusão de Classe III. Apesar de que a Má oclusão não era severa, a queixa principal do paciente era a estética facial, pelo que se optou pela cirurgia ortognática. O planejamento cirúrgico digital foi muito importante para simular os resultados da cirurgia, assim como para a confecção do guia cirúrgico utilizado para a realização das movimentações durante o ato cirúrgico.

\section{REFERÊNCIAS}

1. Furquim L, Sant'ana E, Iwaki L. Tratamento ortodôntico-cirúrgico de um caso de Classe III esquelética agravado pela ausência total dos dentes superiores. Rev Clin Ortod Dental Press. 2002;1(1):23-6.

2. Matos HSA. Anomalia de classe III [dissertation]. Porto (Portugal): Universidade Fernando Pessoa; 2014.

3. Farret MM, Farret MMB, Farret AM. Orthodontic camouflage of skeletal Class III malocclusion with miniplate: a case report. Dental Press J Orthod. 2016;1(4):89-98.

4. Laureano JRL, Carvalho R, Gomes ACA, Bessa RN, Camargo IB. Cirurgia ortognática combinada: relato de um caso. Rev Cir Traumat Buco-Maxilo-Facial. 2002;1(2):31-41.

5. Derton N, Gracco A, Procopio O. Surgical and orthodontic treatment of skeletal Class III featuring severe transversal and sagittal discrepancy. Int Orthod. 2012;10(2):148-64.

6. Park KH, Sandor GK, Kim YD. Skeletal stability of surgery-first bimaxillary orthognathic surgery for skeletal class III malocclusion, using standardized criteria. Int J Oral Maxillofac Surg. 2016;45(9):35-40.

7. Boeck EM. Occurrence of skeletal malocclusions in Brazilian patients with dentofacial deformities. Braz Dent J. 2011;22(4):340-5.

8. Arnett WG, Bergman RT. Facial keys to orthodontic diagnosis and treatment planning. Part I. Am J Orthod Dentofac Orthop. 1993;4(103):299-312.

9. Arslan C. Comparison of the accuracy of manual and digital cephalometric prediction methods in orthognathic surgical planning: a pilot study. Turk J Orthod. 2018;31(4):133-8.

10. Gallego-Romero D, Llamas-Carrera J-M, Torres-Lagares D, Paredes V, Espinar E, Guevara R, et al. Long-term stability of surgicalorthodontic correction of class III malocclusions with long-face syndrome. Med Oral Patol Oral Cir Bucal. 2012;17(3):435-41.

11. Santana E, Janson M. Ortodontia e cirurgia ortognática: do planejamento à finalização. Rev Dental Press Ortod Ortop Maxilar. 2003;8(3):119-29.

12. Cheng T, Hsiu-Hsia L, Lun-Jou L. Intraoral scanning and setting up the digital final occlusion in three-dimensional planning of orthognathic surgery: its comparison with the dental model approach. Plast Reconstr Surg. 2019;143(8):127.

13. Kumar Y. Automated virtual treatment planning in orthodontics: modeling and algorithms [dissertation]. Minneapolis(MN): University of Minnesota; 2012.

14. Choi, SH. Stability of pre-orthodontic orthognathic surgery using intraoral vertical ramus osteotomy versus conventional treatment. J Oral Maxillofac Surg. 2016;74(7):610-9. 